\title{
Extracting Clinical entities and their assertions from Chinese Electronic Medical Records Based on Machine Learning
}

\author{
Jianhong Wang ${ }^{1, a}$, Yousong Peng ${ }^{*}$, , b , Bin Liu' ${ }^{1, c}$, Zhiqiang $\mathrm{Wu}^{1, \mathrm{~d}}$, Lizong \\ Deng $^{2,3, e}$, and Taijiao Jiang ${ }^{*}, 1,2,3, \mathrm{f}$ \\ ${ }^{1}$ College of Computer Science and Electronic Engineering,Hunan University,Changsha,China \\ ${ }^{2}$ Center of System Medicine, Institute of Basic Medical Sciences, Chinese Academy of Medical \\ Sciences \& Peking Union Medical College, Beijing, China \\ ${ }^{3}$ Center of Systems Medicine,Chinese Academy of Medical Sciences,SuZhou,China \\ ajianhong@hnu.edu.cn, bpys2013@hnu.edu.cn, cyjygalb@hnu.edu.cn, dcxiaodiao@hnu.edu.cn, \\ edenglizong@hotmail.com, taijiao@moon.ibp.ac.cn, ${ }^{\star}$ Corresponding author
}

Keywords: Chinese Electronic Medical Records, Information extraction, Named Entity Recognition, assertion classification, Machine Learning

\begin{abstract}
With the rapid growth of electronic medical records (EMRs) in China, large amounts of clinical data have been accumulated. However, limited work for extracting information from EMRs in Chinese has been conducted. In this work, using manually annotated dataset of EMRs in Chinese, we investigated the clinical Named Entities Recognition (NER) based on Conditional Random Field (CRF) and further built a Support Vector Machine (SVM) classifier to determine their assertion status and evaluate the contributions of different features for assertion classification. For Chinese clinical NER, our CRF-based classifier achieved the best F-measure of $89.07 \%$, while the SVM-based assertion classifier achieved a maximum F-measure of $94.10 \%$. Our work suggests that machine learning methods are helpful in NER and assertion determination for Chinese medical clinical records.
\end{abstract}

\section{Introduction}

Electronic Medical Records (EMRs), generated in the process of clinical treatments, refer to systematized collections of patients' clinical information stored in electronic medical records systems [1]. EMRs contain a range of data, including demographics, medical history, medication and allergies, immunization status, laboratory test results, radiology images, vital signs, personal statistics like age and weight, and billing information. A large amount of medical knowledge, closely related to patients, can be discovered through analyzing these medical records [2].

Generally, EMRs are always described in the form of natural language, from which mining patient-related health-care medical knowledge needs applications of information extraction related technologies. NER and assertion classification are the fundamental issues in information extraction of EMRs [2], which not only serves for Clinical Decision Support (CDS) research in medical informatics, but also supports for the user's healthy state modeling and personalized healthcare information services in Consumer Health Informatics. A number of information extraction studies on EMRs in English have been conducted. In 2010, the Center of Informatics for Integrating Biology and the Bedside (i2b2) at Partners Health Care System and Veterans Affairs (VA) Salt Lake City Health Care System organized a challenge in natural-language processing (NLP) for clinical data. Two of the main tasks for the challenge are extracting clinical concepts from natural language text and classifying modification (assertion) made about medical problems [3]. Most clinical NER systems achieved good performance using CRF algorithm for modeling[4-8], while SVM algorithm gets more attention for assertion classification [4-6,9,10].

With the rapid growth of EMRs in China, large amounts of clinical data have been accumulated. However, limited work for extracting information from EMRs in Chinese has been conducted. Lei et al. [11] compared different machine learning algorithms and various types of features for NER in 
Chinese admission notes. Recently, Wu et al. [14] designed a deep learning based NLP systems that could automatically learn useful feature representations from unlabeled corpora through unsupervised learning in Chinese clinical text. Most of work focused on clinical NER [11-14]. Besides, there is a lack of publicly available EMRs in Chinese for medical information extraction research. In this study, we manually annotated a corpus of Chinese typical EMRs and described a machine-learning based system to extract the clinical entities and their assertions. Furthermore, we investigated the contributions of different types of features for assertion classifiers. To the best of our knowledge, this is one of the first extensive studies for Chinese medical entity assertion classification.

\section{Datasets and Annotation}

A corpus of typical EMRs, provided by one Chinese electronic medical records system online (http://www.easyemr.com.cn/activitys/activity-list.aspx?activity_id=32), were collected and collated. This corpus contains 200 internal medicine records. The information which each record includes is shown in Fig. 1.

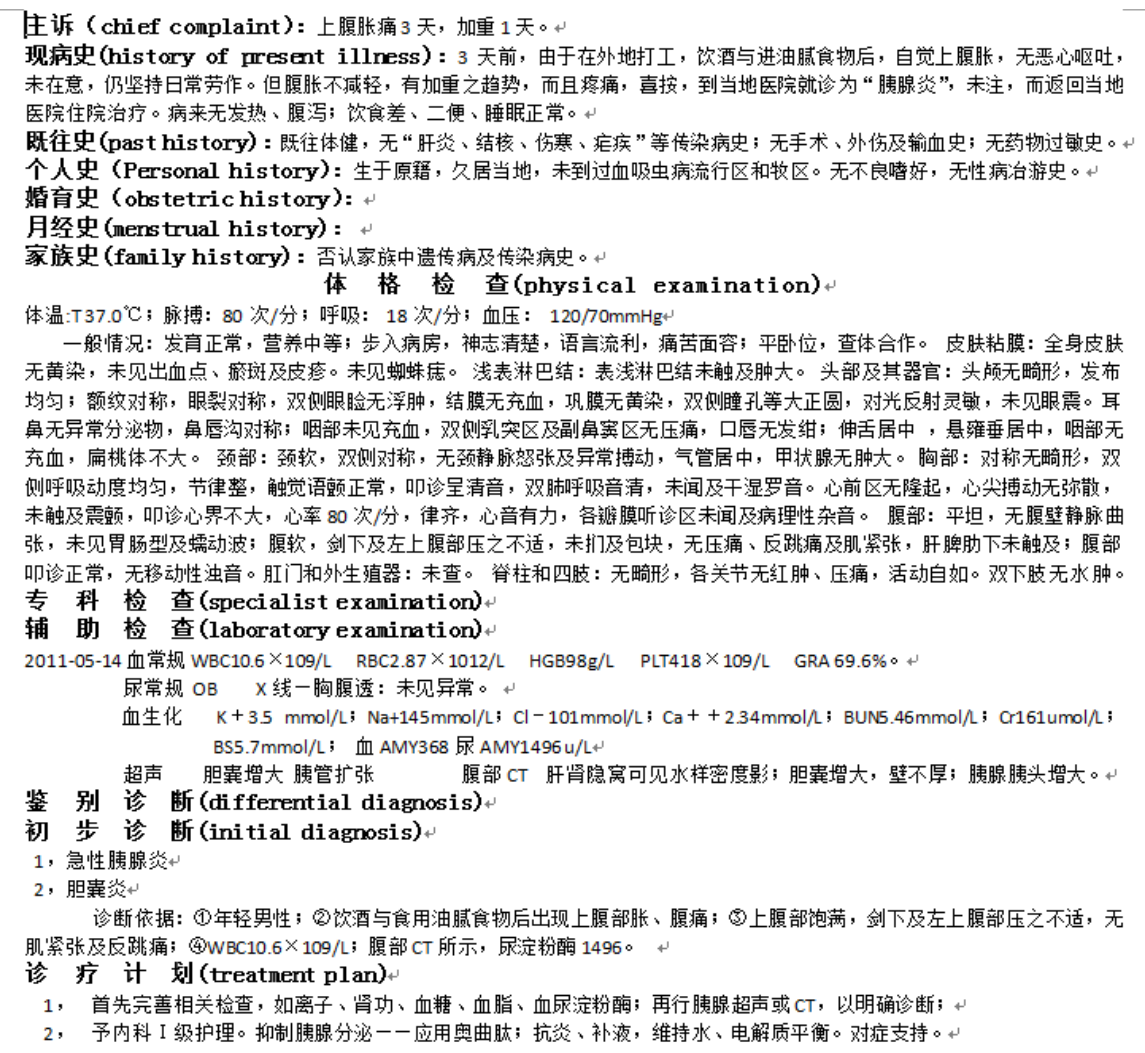

Fig. 1 A sample of a typical Chinese EMRs

Recently, under the guidance of several professional doctors, Yang et al. developed an annotation guideline (http://wi.hit.edu.cn/dev/YuLiao/NER.pdf) for Chinese EMRs according to that of i2b2 [3]. The annotation guideline defines six types of entities, including disease, disease type, complaint-symptom, test-result, test and treatment. For concepts of disease, complaint-symptom, test-result and treatment, eight assertions were further defined: present, absent, possible, conditional, hypothetical, family, history and occasional. Following the above annotation guidelines, two post-graduates of computer science independently marked all the entities and their assertions in our collated EMRs. To calculate the inter-rater agreement for annotation, 40 records were annotated by both annotators. The remaining 160 records were annotated by a single annotator only. The initial agreement between two annotators on 40 records as measured by kappa [15] was 0.926, which indicates the annotation is reliable. 


\section{Methods}

CRF-based approaches for clinical NER. Chinese medical NER task requires determination of the boundaries of clinical entities and assignment of their entity types mentioned above. We converted this NER task to a classification task, for which the CRF algorithm was used (http://crfpp.googlecode.com/svn/trunk/doc/index.html). Firstly, we transformed the annotated data into the 'BIESO' format, in which each word was assigned into a label as follows: $\mathrm{B}=$ beginning of an entity, $\mathrm{I}=$ inside an entity, $\mathrm{E}=$ end of an entity $\mathrm{S}=$ single word entity and $\mathrm{O}=$ outside of an entity. Because the entity type 'disease type' occurred rarely in our collated EMRs, it would be not used in our analyses. Twenty-one tags in total were generated: B-disease, B-complaintsymptom, B-testresult, B-test, B-treatment, I-disease, I-complaintsymptom, I-testresult, I-test, I-treatment, E-disease, E-complaintsymptom, E-testresult, E-test, E-treatment, S-disease, S-complaintsymptom, S-testresult, S-test, S-treatment, and O. Fig. 2 shows a sentence of annotated entities labeled with BIESO tags.

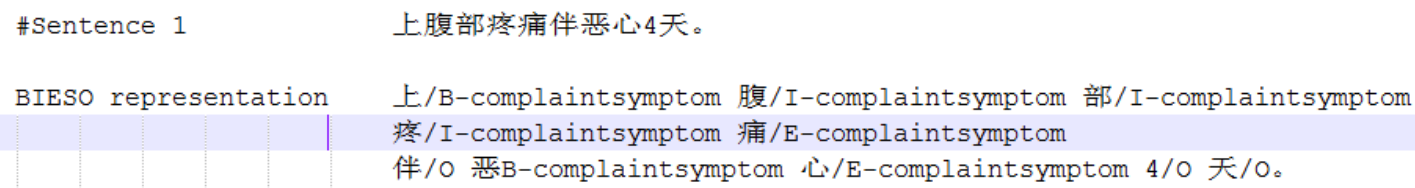

Fig. 2 A sample of Chinese medical NER represented in BIESO format.

Then, we defined the following four types of features to build the CRF classifier for NER task:

1) Single word features: individual Chinese character, punctuation, English alphabet and number.

2) Part of Speech (POS): We use NLRIR (http://ictclas.nlpir.org/), developed by Dr. Zhang Huaping, to get POS tags.

3) Dictionary features: We integrated ICD-10 and Chinese EMRs common terms which contain 3000 phrases into NLPIR. After analyzing concepts of diseases and symptoms appeared in Chinese EMRs, we discovered that most concepts consist of a body part or a subject, such as "体重(weight)", and a basic disease or a symptom such as "减轻 (loss)". Then, a dictionary containing body parts, subjects and basic diseases and symptoms was further built for our CRF-based NER.

4) Section headings: An EMR is a semi-structured narrative text divided into many sections such as chief complaint, present illness and so on. Different sections record different clinical data. For example, the physical examination has concepts of test and abnormal test results, while the section of preliminary diagnosis contains disease concepts. Therefore, we manually reviewed some records and defined 12 different section headings as section headings feature.

SVM-based approaches for assertion classifier. Assertion classification is to assign one of the eight possible assertion labels mentioned above to the entity type of disease, complaint-symptom, test-result, test and treatment. The SVM algorithm was used to conduct the classification, which was implemented using libsvm (http://www.csie.ntu.edu.tw/ cjlin/libsvm/) with a linear kernel. Four types of features were used as follows:

1) Context feature: $a+4$ word window, i.e., words that appear within $t+4$ word window of the target concept. Given the concept at the $n$th position in the sentence, the $\pm \overline{4}$ word window captures the words found in the $(\mathrm{t}-1) \mathrm{th},(\mathrm{t}-2) \mathrm{th},(\mathrm{t}-3) \mathrm{th},(\mathrm{t}-4) \mathrm{th},(\mathrm{t}+1) \mathrm{th},(\mathrm{t}+2) \mathrm{th},(\mathrm{t}+3) \mathrm{th}$, and $(\mathrm{t}+4)$ th positions in the sentence;

2) Section headings: 12 different section headings were used as mentioned above.

3) Cues information feature: before or after a target concept, some word cues can indicate the target concept is present or absent. For example, in the sentence of “双侧眼睑无浮肿 (no bilateral eyelid edema)", ”浮肿(edema)” is a target concept, and “无(no)” before “浮肿(edema)” indicates “浮 肿(edema)" is absent for patient. Then, we developed a system to automatically extract these cues.

4) POS features.

The above features were transformed into binary features. For each feature, the binary feature vector lists all possible values of that feature in the corpus as its columns, and for each target medical problem to be classified (row), it sets the columns observed to 1, leaving the rest at zero. If the target has no value for a feature, then all columns representing this feature will be set to zero. 
Experiments and evaluation. The internal medicine typical records mentioned above were divided into two subsets: two-thirds ( 120 records) for training and one-third ( 80 records) for test. The parameters of CRF classifier and SVM classifier were optimized using the training set via the five-fold cross-validations. Then we tested the classifiers using the independent test set. The precision, recall and F-measure were used to measure the performance of the classifiers. To evaluate the contributions of different types of features for assertion classification, we started with the context feature firstly, and then added additional types of features and reported corresponding results.

\section{Results and Discussion}

Table 1 describes the statistics of entities in the annotated EMRs used in our study. There are 13119 entities in the training dataset and 8026 entities in the test dataset. Distribution of different types of entities' assertions in the training set and test set were shown in Table 2 . The proportion of present, absent, possible and history assertions is far larger than other assertion types. In our annotated dataset, the number of hypothetical, conditional and family assertion is lower than 20 , so we ignore these assertion types.

Table 1 Distribution of different types of entities in training set and test set

\begin{tabular}{lcccccc}
\hline \multicolumn{1}{r}{ Data set } & disease & complaintsymptom & testresult & test & treatment & All \\
\hline training & 1403 & 1623 & 5362 & 2086 & 845 & 11319 \\
test & 1031 & 976 & 3786 & 1620 & 613 & 8026 \\
total & 2434 & 2599 & 9148 & 3706 & 1458 & 19345 \\
\hline
\end{tabular}

Table 2 Distribution of different types of assertions in training set and test set

\begin{tabular}{lcccccc}
\hline \multicolumn{1}{c}{ Data set } & Present & absent & possible & occasional & history & All \\
\hline training & 1921 & 6146 & 527 & 26 & 175 & 8795 \\
test & 1395 & 4143 & 371 & 20 & 110 & 6039 \\
total & 3316 & 10289 & 898 & 46 & 285 & 14834 \\
\hline
\end{tabular}

The detailed results of CRF classifier for each entity type were shown in Table 3. F-measures ranged from $78.47 \%$ to $94.58 \%$ among five types of entity. Performance was best for the type "test" and worst for the type "treatment". For each type of entity, precision was higher than recall.

Table 3 Result of CRF-based named entity recognition for each entity

\begin{tabular}{|c|c|c|c|}
\hline Entity type & Precision & recall & F-measure \\
\hline disease & $90.22 \%$ & $82.66 \%$ & $86.27 \%$ \\
\hline complaint-symptom & $87.24 \%$ & $78.40 \%$ & $82.58 \%$ \\
\hline test-result & $94.65 \%$ & $94.51 \%$ & $94.58 \%$ \\
\hline test & $84.85 \%$ & $82.77 \%$ & $83.80 \%$ \\
\hline treatment & $92.90 \%$ & $67.92 \%$ & $78.47 \%$ \\
\hline overall & $91.31 \%$ & $86.94 \%$ & $89.07 \%$ \\
\hline
\end{tabular}

Our best performance results are similar to the results in [13] for discharge summary and the NER task in i2b2 2010 challenge [3, 8]. In this study, we found that our CRF model could correctly identify the entity that did not appear in the training set. The reason may mainly lie in the dictionary features used in our study. In Chinese EMRs, most entities have their syntactic structures. For example, the concept of "左侧肢体(left limb)无力(weakness)" is made up of a body part "左侧肢体(left limb)" and a basic symptom "无力(weakness)". In our dictionary, we defined these common body parts and basic symptoms and diseases. Table 4 shows the performance of SVM classifier in assertion determination when using different feature sets. Context feature set was observed to contribute most to assertion classification with the F-measure as high as $92.07 \%$. Both section headings feature and cues information feature improved assertion classifier performance. For example, section headings 
feature improved F-measure from $92.07 \%$ to $93.14 \%$, while cues information improved F-measure from $92.07 \%$ to $93.56 \%$. POS did not further improve assertion classification performance.

Table 4 Results of SVM-based assertion classifier when different sets of features were used

\begin{tabular}{lc}
\hline \multicolumn{1}{c}{ Feature set } & F-measure \\
\hline context feature & $92.07 \%$ \\
context feature+section headings & $93.14 \%$ \\
context feature+POS & $92.18 \%$ \\
context feature+cues information & $93.56 \%$ \\
context feature+section headings+POS+cues information & $94.10 \%$ \\
\hline
\end{tabular}

Table 5 Detailed results of the best SVM-based Assertion classifier for each entity type

\begin{tabular}{|c|c|c|c|}
\hline Assertion category & Precision & recall & F-measure \\
\hline present & $95.68 \%$ & $92.95 \%$ & $94.30 \%$ \\
\hline absent & $96.93 \%$ & $93.62 \%$ & $95.25 \%$ \\
\hline occasional & $92.65 \%$ & $89.51 \%$ & $91.05 \%$ \\
\hline possible & $78.86 \%$ & $63.47 \%$ & $70.33 \%$ \\
\hline history & $81.31 \%$ & $74.37 \%$ & $77.69 \%$ \\
\hline overall & $95.06 \%$ & $93.15 \%$ & $94.10 \%$ \\
\hline
\end{tabular}

The detailed results of the best SVM assertion classifier for each assertion types are shown in Table 5.The overall F-measure is $94.10 \%$.The classifier achieved the best performances for the present and absent assertion, with F-measure equaling to $94.30 \%$ and $95.25 \%$, while it performed worst for the possible assertion (F-measure $=70.33 \%)$. This may be due to the fact that human determination of 'possible' assertions was not always straightforward, as reported by Jiang's work [3].

\section{Conclusion}

In this study, we investigated clinical NER from EMRs in Chinese based on CRF algorithm and further built a SVM-based classifier to determine assertion status and evaluate the effects of different features for assertion classification. For the former, our CRF classifier achieved the best F-measure of $89.07 \%$, while for the latter our SVM assertion classifier get a maximum F-measure of 94.10\%. Although our experiment achieved a good performance, there are still some limitations. The data used in our study were limited to internal medicine records. In the future, we will cooperate with hospitals to get more medical records to improve our models. Overall, our work suggests machine-learning methods could be helpful in NER and assertion determination for the Chinese EMRs.

\section{Acknowledgements}

This study was supported by National Natural Science Foundation (31500126 and 31371338), and the Young Teachers Development Plan of Hunan University to YS (531107040720) and the International Scientific and Technological Cooperation project (2014DFB30010). We would like to thank the members of the Jiang lab for their help and deliberations. We would also like to thank the anonymous reviewers for their valuable comments and suggestions. No competing interests exist.

\section{References}

[1] Information on http://www.nhfpc.gov.cn.

[2] Yang Jinfeng, Yu Qiubin, Guan Yi, Jiang ZhiPeng: An Overview of Research on Electronic Medical Record Oriented Named Entity Recognition and Entity Relation Extraction. Acta Automatica Sinica. Vol. 40 (2014), p. 1537-1562. 
[3] Özlem Uzuner, South B R, Shen S, Duvall S L: 2010 i2b2/VA challenge on concepts, assertions, and relations in clinical text. J Am Med Inform Assoc. Vol. 18 (2011), p. 552-556.

[4] Jiang M, Chen Y, Liu M, Rosenbloom ST, Mani S, Denny JC and Xu H: A study of machine-learning-based approaches to extract clinical entities and their assertions from discharge summaries. J Am Med Inform Assoc. Vol. 18 (2011), p. 601-606.

[5] Bruijn B D, Cherry C, Kiritchenko S, Martin J and Zhu X: Machine-learned solutions for three stages of clinical information extraction: the state of the art at i2b2 2010. J Am Med Inform Assoc. Vol. 18 (2011), p. 557-62.

[6] Grouin C, Abacha A B, Bernhard D, CARAMBA: concept, assertion, and relation annotation using machine-learning based approaches. In: Proceedings of the 2010 I2B2/VA Workshop on Challenges in Natural Language Processing for Clinical Data. Boston, MA, USA (2010).

[7] Uzuner O, Solti I, Cadag E: Extracting medication information from clinical text. J Am Med Inform Assoc. Vol. 17 (2010), p. 514-518.

[8] Doan S, Collier N, Hua X, Duy P H and Tu M P: Recognition of medication information from discharge summaries using ensembles of classifiers. Bmc Medical Informatics \& Decision Making. Vol. 12 (2012), p. 1-10.

[9] Uzuner O, Zhang X, Sibanda T: Machine learning and rule based approaches to assertion classification. J Am Med Inform Assoc. Vol. 16 (2009), p. 109-115.

[10] Clark C, Aberdeen J, Coarr M, Tresner-Kirsch D, Wellner B, Yeh A, Hirschman L: MITRE system for clinical assertion status classification. J Am Med Inform Assoc. Vol. 18 (2011), p. 563-7.

[11]Lei J, Tang B, Lu X, Gao K, Min J, Hua X: A comprehensive study of named entity recognition in Chinese clinical text. J Am Med Inform Assoc. Vol. 21 (2014), p. 808-14.

[12] Wang, Shi Kun, L. I. Shao zi, and T. S. Chen: Recognition of Chinese Medicine Named Entity Based on Condition Random Field. Journal of Xiamen University. Vol. 48 (2009), p. 359-364.

[13] YAN yang, WEN Dun-wei, WANG Yun-ji, WANG Ke: Named entity recognition in Chinese medical records based on cascaded conditional random field. Journal of Jilin University (Engineering and Technology Edition). Vol. 44 (2014), p. 1843-1848.

[14] Wu Y, Jiang M, Lei J, Xu H: Named Entity Recognition in Chinese Clinical Text Using Deep Neural Network. Studies in Health Technology \& Informatics. Vol. 216 (2015), p. 624-628.

[15] Hripcsak, George, and A. S. Rothschild: Agreement, the F-Measure, and Reliability in Information Retrieval. J Am Med Inform Assoc. Vol. 12 (2005), p. 296-298. 